\title{
HOLONOMY DECOMPOSITIONS OF NEAR-RINGS
}

\author{
by M. HOLCOMBE \\ (Received 15th September 1978)
}

Some techniques developed by Eilenberg (1) in the theory of automata are used to investigate certain types of near-ring.

\section{Transformation semigroups}

Let $P$ be a non-empty set and $S$ a semigroup with identity then the pair $(P, S)$ is called a transformation semigroup if a semigroup homomorphism $\phi: S \rightarrow$ Map $P$ exists with a trivial kernel. For the sake of notational clarity we will write the action of an element $s \in S$ on an element $p \in P$ as $s(p)$, rather than $\phi(s)(p)$. For any $p \in P$ define $\bar{p}: P \rightarrow\{p\}$ as the constant transformation of $p$ and then put $(\overline{P, S})=$ $\left(P,\left\langle S \cup \cup \cup_{p \in P}\{\bar{p}\}\right\rangle\right)$, where $\left\langle S \cup \cup_{p \in P}\{\bar{p}\}\right\rangle$ is the semigroup generated by $S$ and $\cup_{p \in P}\{\bar{p}\}$. For any $s \in S$ the set $s(P)=\{s(p) \mid p \in P\}$ will be called the $s$-image of $P$. Associated with $(P, S)$ is the space $\mathscr{J}^{0}$ of all $s$-images of $P$. Note that $\{p\}$ may not belong to $\mathscr{J}^{0}$ where $p \in P$. Thus we introduce the idea of the skeleton space $\mathscr{F}$ of $(P, S)$, this consists of $\mathscr{J}^{0} \cup P \cup\left(\cup_{p \in P}\{\{p\}\}\right)$. A preorder $\leqslant$ may be defined on $\mathscr{g}$ as follows, if $A, B \in \mathscr{J}$ then $A \leqslant B$ iff $A \subseteq s(B)$ for some $s \in S$. The set $\mathscr{J}$ with the ordering $\leqslant$ is called the skeleton of $(P, S)$. We may now define an equivalence relation $\sim$ on $\mathscr{J}$ by putting $A \sim B$ iff $A \leqslant B$ and $B \leqslant A$. We can now introduce the holonomy groups.

Let $A \in \mathscr{J}^{0}$ and put $K(A)$ to be the set of elements of $S$ that behave as units on $A$, more precisely $K(A)=\{s \in S \mid \exists t \in S$ with $s A=A$ and $s t(a)=t s(a)=a, \forall a \in A\}$. Note that for a given $s \in S$ there may exist more than one $t$ which acts as an inverse for $s$ on $A$. However the restriction of $t$ to $A$ is unique. Let $B(A)=\{B \in \mathscr{J} \mid B \subseteq A$ and if $C \in \mathscr{J}$ with $B \subseteq C \subseteq A$ then either $C=B$ or $C=A\}$. Thus $B(A)$ consists of those images of $P$ that are contained in $A$ and are maximal with respect to this property. Each $s \in K(A)$ acts as a permutation on $B(A)$ and the set $\mathscr{G}(A)$ of the distinct permutations of $B(A)$ induced by the elements of $K(A)$ is called the holonomy group of $A$. Then the group $\mathscr{G}(A)$ acts as a transformation group on the maximal image space of $A, B(A)$. Eilenberg proved that any finite transformation semigroup may be covered by a wreath product formed from these holonomy transformation groups. This insistence on the finiteness of both $P$ and $S$ is not entirely essential. The notion of a covering may also be extended to other situations. Let $(P, S)$ and $(Q, T)$ be transformation semigroups. We say that $(Q, T)$ covers $(P, S)$, written $(Q, T) \geqslant(P, S)$ if there exists an onto partial function $f: Q \rightarrow P$ and if to each $s \in S$ there corresponds a $t \in T$ satisfying $s(f(q))=f(t(q))$ for $q \in Q$.

The wreath product of two transformation semigroups $(P, S) \circ(Q, T)$ consists of 
the pair $\left(P \times Q, S \times T^{p}\right)$ where $T^{p}$ is the set of all maps from $P$ into $T$ and we define $(s, h)(p, q)=(s(p),(h(p))(q))$ for all $(s, h) \in S \times T^{p},(p, q) \in P \times Q$. Returning to the skeleton $(\mathscr{F}, \leqslant)$ of $(P, S)$ we now examine the concept of a height function. This is a function $h$ from $\mathscr{J}$ into the set $Z$ of integers satisfying the conditions

H.F.1 $h(\{p\})=0$ for all singletons $\{p\} \in \mathscr{J}$

H.F.2 $A \sim B \Rightarrow h(A)=h(B)$ where $A, B \in \mathscr{J}$

H.F.3 $A<B \Rightarrow h(A)<h(B)$ if $A, B \in \mathscr{J}$ and card $A>1$

H.F.4 If $0 \leqslant i \leqslant h(P)$ then $\exists A \in \mathscr{J}$ such that $h(A)=i$.

There may be many height functions on $(P, S)$ and if $(P, S)$ is finite, height functions always exist. In the rest of this section $h: \mathscr{J} \rightarrow Z$ will denote a fixed height function and $h(P)$ will be called the height of $(P, S)$.

Let $0 \leqslant i \leqslant h(P)$ and write $\mathscr{I}(i)$ for the set of all elements of $\mathscr{J}$ of height $i$, so $\mathscr{J}(i)=\{A \in \mathscr{I} \mid \boldsymbol{h}(A)=i\}$. We may partition $\mathscr{F}(i)$ using the equivalence relation defined on $\mathscr{I}$. If there are a finite number of equivalence classes under $\sim$ on the set $\mathscr{I}(i)$ for each $0 \leqslant i \leqslant h(P)$, we say that $(P, S)$ is of finite breadth. Given any $0 \leqslant i \leqslant$ $h(P)$ we choose a set $\left\{A_{i_{j}} \mid j \in J\right\}$ of representatives of the equivalence classes on $\mathscr{J}(i)$ and then define the transformation semigroup $\left(\Pi_{j \in J} B\left(A_{i_{j}}\right), \Pi_{j \in J} \mathscr{G}\left(A_{i_{j}}\right)\right)$ which will be denoted by the symbol $\mathscr{H}_{i}$. The result of Eilenberg's that we need is:

Theorem A. If $(P, S)$ is a finite transformation semigroup and $h: \mathscr{J} \rightarrow Z$ is a height function then $(P, S) \leqslant \overline{\mathscr{H}}_{n} \circ \overline{\mathscr{H}}_{n-1} \circ \ldots \circ \overline{\mathscr{H}}_{1}$ where $n=h(P)$.

Thus the semigroup $S$ is a homomorphic image of subsemigroup of a wreath product of groups formed from the distinct holonomy groups. If we move to the case where $(P, S)$ is an infinite transformation semigroup then the result may still be true. The wreath product of an infinite family of transformation groups may be defined but Eilenberg's proof, which involves the use of relational coverings of finite rank, does not seem to generalize very easily. However if the iransformation semigroup has a finite height function and is of finite breadth then we may make some progress.

We will apply the Eilenberg techniques in one main direction.

\section{The 2-primitive near-ring $M_{G}(\Gamma)$.}

In this section $N=M_{G}(\Gamma)$ where $G$ is a group of automorphisms on $\Gamma$, an additive group, and the number of orbits of $\Gamma$ under $G$ is $n<\infty$. The near-ring $N$ becomes a transformation semigroup $(N, N)$ when we interpret the operation of the semigroup of $N$ on $N$ as left multiplication. The set $\mathscr{J}^{0}$ then consists of all the principal right $N$-groups of $N$ of the form $a N, a \in N$.

Suppose that $\gamma_{1}, \ldots, \gamma_{n}$ are distinct non-zero orbit representatives, so that $\Gamma=$ (0) $\cup\left(\cup \cup_{i=1}^{n} \gamma_{i} G\right)$. Put $I=\{1,2, \ldots, n\}$, then for each $i \in I$ there exists an idempotent $e_{i} \in N$ defined by $\gamma_{i} e_{i}=\gamma_{i}$ and $\gamma_{j} e_{i}=0=0 e_{i}$ for $i \neq j$. Then $N=\oplus_{i \in I} e_{i} N$. The $N-$ subgroups $e_{i} N$ are minimal (type 2) right ideals of $N$. Note that $e_{i} N \in \mathscr{J}$ for $i \in I$. Now 
let $J \subseteq I$ and consider $\oplus_{j \in J}\left(e_{j} N\right)$. Since $\Sigma_{j \in J} e_{j} \in \oplus_{j \in J} e_{j} N$ we have $\left(\Sigma_{j \in J} e_{j}\right) . N \subseteq$ $\bigoplus_{i \in J}\left(e_{j} N\right)$. Now let $\Sigma_{j \in J} e_{j} n_{i} \in \oplus_{j \in J}\left(e_{i} N\right)$ where $n_{j} \in N$. Define elements $m_{i} \in N$ for $j \in J$ by $\gamma_{j} m_{j}=\gamma_{j} n_{j}, \gamma_{k} m_{j}=0$ for $k \neq j, k \in I$. Consider $\left(\sum_{j \in J} e_{j}\right)\left(\sum_{j \in J} m_{j}\right)$ then for

$$
i \in I, \gamma_{i}\left(\sum_{j \in J} e_{j}\right)\left(\sum_{i \in J} m_{j}\right)=\left\{\begin{array}{c}
0 \text { if } i \notin J \\
\gamma_{i} n_{i} \text { if } i \in J
\end{array}\right.
$$

Now

$$
\gamma_{i}\left(\sum_{j \in J} e_{j} n_{j}\right)=\left\{\begin{array}{cc}
0 & \text { if } i \notin J \\
\gamma_{i} n_{i} & \text { if } i \in J
\end{array}\right.
$$

and so

$$
\sum_{j \in J} e_{j} n_{j}=\left(\sum_{j \in J} e_{i}\right)\left(\sum_{j \in J} m_{j}\right) \in\left(\sum_{j \in J} e_{j}\right) N
$$

hence $\oplus_{i \in J}\left(e_{j} N\right)=\left(\Sigma_{j \in J} e_{j}\right) N$. Thus each $\oplus_{i \in J}\left(e_{i} N\right) \in \not{J}$ for $J \subseteq I$. Also, for $J \subseteq I$ and $J^{\prime} \subseteq I$ with $|J|=\left|J^{\prime}\right|$ we have $\left(\sum_{j \in J} e_{j}\right) N \sim\left(\sum_{k \in J^{\prime}} e_{k}\right) N$. To see this we introduce the following mappings, for $t, s \in I$ define $f_{r s}: \Gamma \rightarrow \Gamma$ by $\gamma_{r} f_{r s}=\gamma_{s}, \gamma_{t} f_{r s}=0$ for $t \neq r$ and $0 f_{r s}=0$. Then $f_{r s} \in N$. Now let $J=\left\{j_{1}, j_{2}, \ldots, j_{q}\right\}$ and $J^{\prime}=\left\{k_{1}, k_{2}, \ldots, k_{q}\right\}$ and consider $y=\left(\sum_{t=1}^{q} f_{i_{t} k_{t}}\right)\left(\sum_{i=1}^{q} e_{k_{t}}\right)\left(\sum_{t=1}^{q} f_{k_{t_{i}}}\right)$. Then $\gamma_{i_{t}} y=\gamma_{i_{t}}$ for $t=1, \ldots, q$ and $\gamma_{i} y=0$ for $i \notin J$. Thus $y=\sum_{i=1}^{q} e_{i_{t}}=\sum_{j \in J} e_{j}$ and thus $\left(\sum_{j \in J} e_{j}\right) N \subseteq\left(\sum_{t=1}^{q} f_{j_{t} k_{t}}\right)\left(\sum_{k \in J} e_{k}\right) N$ and so $\left(\sum_{j \in J} e_{j}\right) N \leqslant$ $\left(\Sigma_{k \in J^{\prime}} e_{k}\right) N$ and by symmetry $\left(\sum_{j \in J} e_{j}\right) N \sim\left(\Sigma_{k \in J} e_{k}\right) N$. Also $\sum_{j \in J} e_{j} N=$ $\left(\sum_{i=1}^{q} f_{i k_{k}}\right) \Sigma_{k \in J^{\prime}} e_{k} N$.

Clearly if $J \subseteq J^{\prime \prime} \subseteq I$ then

$$
\left(\sum_{j \in J} e_{j}\right) N \leqslant\left(\sum_{i \in J^{n}} e_{i}\right) N
$$

We now consider any $0 \neq a \in N$ and look at the principal right $N$-group $a N$. Clearly $a N \in \mathscr{J}$. Suppose that there are $s$ distinct non-zero orbits in the set $\Gamma a$, where $1 \leqslant s \leqslant n$, let these have representatives $\gamma_{i_{1}}, \ldots, \gamma_{i_{s}}$. Put $\vec{e}_{s}=\sum_{m=1}^{s} e_{i_{m}}$ then $a \bar{e}_{s}=a$ for if $\gamma \in \Gamma$ then $\gamma a=\gamma_{i_{m}} g$ for some $m \in\{1, \ldots, s\}$ and $g \in G$, then $\gamma a \bar{e}_{s}=\gamma_{i_{m}} g \bar{e}_{s}=$ $\gamma_{i_{m}} \bar{e}_{s} g=\gamma_{i_{m}} g=\gamma a$. Hence $a N \subseteq a \bar{e}_{s} N$ and so $a N \leqslant \bar{e}_{s} N$. Now, for each $m \in$ $\{1, \ldots, s\} \exists j_{m} \in I$ such that $\gamma_{j_{m}} a=\gamma_{i_{m}} g_{m}$ for some $g_{m} \in G$. Define a function $n^{\prime}: \Gamma \rightarrow \Gamma$ by $\gamma_{i_{m}} n^{\prime}=\gamma_{i_{m}}$ for $m \in\{1, \ldots, s\}$ and $\gamma n^{\prime}=0$ for $\gamma \in \Gamma \backslash \cup_{m=1}^{s} \gamma_{i_{m}} G$. Then $n^{\prime} \in N$. Also define for each $m \in\{1, \ldots, s\}$ a map $n_{i_{m}}: \Gamma \rightarrow \Gamma$ by $\gamma_{i_{m}} n_{i_{m}}=\gamma_{i_{m}} g_{m}^{-1}$ where $g_{m} \in G$ and $\gamma_{j_{m}} a=\gamma_{i_{m}} g_{m}$. Now consider $z=n^{\prime} a .\left(\sum_{m=1}^{s} n_{i_{m}}\right)$. Let $l \in\{1, \ldots, s\}$ then $\gamma_{i l} z=\gamma_{i l} a\left(\sum_{m=1}^{s} n_{i_{m}}\right)$ where $\gamma_{i l} a=\gamma_{i,} g_{l}$, so $\gamma_{i_{i}} z=\gamma_{i} g_{l}\left(\sum_{m=1}^{s} n_{i_{m}}\right)=\gamma_{i_{r}}$. For $\gamma \in \Gamma \mid \sum_{i=1}^{s} \gamma_{i l} G$, $\gamma_{z}=0$. Thus $z=\bar{e}_{s}$ and hence $\bar{e}_{s} N \subseteq n^{\prime} a N \Rightarrow \bar{e}_{s} N \leqslant a N$. Thus $\bar{e}_{s} N \sim a N$. Note that $\bar{e}_{s} N \subseteq \bar{e}_{s} n^{\prime} a N \subseteq \bar{e}_{s} N$ so $\bar{e}_{s} N=\left(\bar{e}_{s} n^{\prime}\right) a N$ and also $a N=a \bar{e}_{s} N$.

Theorem 1. For $a \in N$ with $a \neq 0$ then $a N \sim \bar{e}_{s} N$ where $\bar{e}_{s}$ is the sum of $s$ distinct idempotents where $s$ is the number of non-zero orbits in $\Gamma a$.

This means that the skeleton of $M_{G}(\Gamma)$ is particularly simple. The height of $a N$ is the number of non-zero orbits of $\Gamma a$ and $M_{G}(\Gamma)$ has a maximum breadth of 1 . 
Thus we have:-

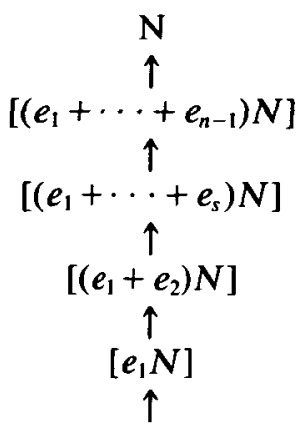

(0)

where the square brackets indicate -equivalence classes and the vertical arrows denote the relationship $<$.

We must now establish the holonomy groups of the rt. $N$-subgroups $\bar{e}_{s} N=$ $\left(e_{1}+\cdots+e_{s}\right) N$. Recall that $K\left(\bar{e}_{s} N\right)=\left\{a \in N \mid \exists \bar{a} \in N\right.$ with $a \bar{e}_{s} N=\bar{e}_{s} N$ and $\bar{a} a e_{s} n=$ $a \bar{a} e_{s} n=e_{s} n$ for all $\left.n \in N\right\}$.

Theorem 2. $a \in K\left(\bar{e}_{s} N\right)$ if and only if $\left(\Gamma \bar{e}_{s}\right) a=\Gamma \bar{e}_{s}$ and $a$ is bijective on $\left(\Gamma \bar{e}_{s}\right)$.

Proof. If $\left(\Gamma \bar{e}_{s}\right) a=\Gamma \bar{e}_{s}$ with $a$ bijective on $\Gamma \bar{e}_{s}$, let $\gamma_{i} a=\gamma_{j} g_{i}$, where $j_{i} \in\{1, \ldots, s\}$ for $i=1, \ldots, s$. Define $\bar{a}: \Gamma \rightarrow \Gamma$ by $\gamma_{j i} \bar{a}=\gamma_{i} g_{i}^{-1}$ for $i=1, \ldots, s, 0 \bar{a}=0$ and $\gamma \bar{a}=0$ for $\gamma \in \Gamma \backslash \Gamma \bar{e}_{s}$. Then $\gamma_{p} a \bar{a}=\gamma_{p}$ for $p \in\{1, \ldots, s\}$ and $\gamma_{p} \bar{a} a=\gamma_{p}$ for $p \in\{1, \ldots, s\}$. Then $\gamma_{p} a \overline{a e}_{s} n=\gamma_{p} \bar{e}_{s} n=\gamma_{p} \bar{a} a \bar{e}_{s} n$ for all $p \in\{1, \ldots, s\}$. For $j \notin\{1, \ldots, s\}$ then $\gamma_{j} a \bar{a}_{s} n=0=$ $\gamma_{j} \bar{a} a \bar{e}_{s} n=\gamma_{j} \bar{e}_{s} n$. Hence $a \in K\left(\bar{e}_{s} N\right)$. The converse is also easily proved.

We now construct the set $B\left(\bar{e}_{s} N\right)$. Let $\underline{S}=\{1,2, \ldots, s\}$ and $\bar{S}^{\circ}=\{0,1,2, \ldots, s\}$. Suppose that $a N \in B\left(\bar{e}_{s} N\right)$ then $a N \subset_{\neq} \bar{e}_{s} N$ and if $a N \subseteq b N \subseteq \bar{e}_{s} N$ for some $b \in N$ then either $a N=b N$ or $b N=\bar{e}_{s} N$. Clearly $a \in \bar{e}_{s} N$ and so $\gamma_{j} a=0$ for $j \in I \backslash \underline{S}$. We examine $\gamma_{i} a$ for $i \in \underline{S}$. It is soon evident that either $\gamma_{i} a$ is non-zero for all $i \in \underline{S}$ or $\gamma_{i} a=0$ for only one $i \in \underline{S}$, otherwise $a N$ ceases to be maximal in $\bar{e}_{s} N$. We have two cases, in the first case there exists $i, j \in \underline{S}$ such that $\gamma_{i} a \sim \gamma_{j} a$ and the remaining $\gamma_{k} a$ are in distinct orbits, and in the second case $\gamma_{i} a=0$ for one $i \in \underline{S}$ and in distinct orbits for all other $\gamma_{k} a$. We now represent $a$ by the pair $(f, h)$ where $f: \underline{S}^{\circ} \rightarrow \underline{S}^{\circ}, h: \underline{S} \rightarrow G$ so that $\gamma_{i} a=\gamma_{f(i)} h(i)$ where $f(0)=0$ and $\gamma_{0}$ is interpreted as $0_{\Gamma}$. Then in either case $\left|f\left(\underline{S}^{\circ}\right)\right|=s$ and any such pair will define an element $a$ such that $a N$ is maximal as a $r$. $N$-group in $\bar{e}_{s} N$. However $a N=b N$ could occur with $a \neq b$. To find the distinct elements of $B\left(\bar{e}_{s} N\right)$ we must consider the relation $a \approx b \Leftrightarrow a N=b N$. Then if $a=(f, h), b=\left(f_{1}, h_{1}\right)$ we have $a \in b N$ and $b \in a N$. Then $\exists n, n^{\prime} \in N$ such that $a=b n, b=a n^{\prime}$. Thus for $i \in \underline{S}$

$$
\gamma_{i} a=\gamma_{i} b_{n} \text {, that is } \gamma_{f(i)} h(i)=\gamma_{f^{\prime}(i)} h^{\prime}(i) n=\gamma_{f^{\prime}(i)} n h^{\prime}(i) \text {. }
$$

If $f(i)=0$ but $f(j) \neq 0$ for $j \in \underline{S} \backslash\{i\}$ then $\left(e_{1}+\cdots \uparrow^{i} \cdots e_{s}\right) a=a$ and so $a \in$ $\left(e_{1}+\cdots \uparrow i \cdots+e_{s}\right) N$ which is maximal in $\left(e_{1}+\cdots+e_{s}\right) N$. However, if $f^{\prime}(i)=f^{\prime}(j) \neq 0$ for $i \neq j$ then $\gamma_{f^{\prime}(i)} n=\gamma_{f(i)} h(i)\left(h^{\prime}(i)\right)^{-1}$ and $\gamma_{f^{\prime}(i)} n=\gamma_{f(j)} h(j)\left(h^{\prime}(j)\right)^{-1}$ which implies that $f(i)=f(j)$ and $h(i)\left(h^{\prime}(i)\right)^{-1}=h(j)\left(h^{\prime}(j)\right)^{-1}$. 
Thus $(f, h) \approx\left(f^{\prime}, h^{\prime}\right) \Leftrightarrow$ either $f(i)=f^{\prime}(i)=0$ for some $i \in \underline{S}$ :

$$
\begin{aligned}
& \text { or } f(i)=f(j) \neq 0 \Leftrightarrow f^{\prime}(i)=f^{\prime}(j) \neq 0 \\
& \text { and } h(i)\left(h^{\prime}(i)\right)^{-1}=h(j) .\left(h^{\prime}(j)\right)^{-1} .
\end{aligned}
$$

Then $B\left(\bar{e}_{s} N\right)$ consists of the $\approx$-equivalence classes constructed in this way. The elements of $K\left(\bar{e}_{s} N\right)$ gives rise to mappings $\hat{a}: \Gamma \bar{e}_{s} N \rightarrow \Gamma \bar{e}_{s} N$ which are bijective on $\Gamma \bar{e}_{s} N$ and each map $\hat{a}$ may be considered to be an ordered pair $\left(\hat{a}^{*}, \hat{a}^{* *}\right)$ where $\hat{a}^{*}: S \rightarrow S$ and $\hat{a}^{* *}: \underline{S} \rightarrow G$ defined by $\hat{a}^{*}(i)=j_{i}$ and $\hat{a}^{* *}(i)=g_{i}$ where $\gamma_{i} \hat{a}=\gamma_{j} g_{i}$ for each $\bar{i} \in \bar{S}$. Thus we need only look at the group $\left(S_{S}\right) \circ G$. We define the relation $\equiv$ on $\left(S_{S}\right) \circ G$ by putting $(\alpha, \beta) \equiv\left(\alpha_{1}, \beta_{1}\right) \Leftrightarrow(\alpha, \beta) x N=\left(\alpha_{1}, \beta_{1}\right) x N$ for all $x N \in B\left(\bar{e}_{s} N\right)$. Now let $x=$ $(f, h)$ where $f: \underline{S}^{\circ} \rightarrow \underline{S}^{\circ}$ with $\left|f\left(\underline{S}^{\circ}\right)\right|=s$ and $h: \underline{S} \rightarrow G$ as before. Then we want $(\alpha, \beta)$ $(f, h) \approx\left(\alpha_{1}, \beta_{1}\right)(\bar{f}, h)$ for all $(f, h)$ of this type i.e. $(\bar{f}(\alpha), h(\alpha) \cdot \beta) \approx\left(f\left(\alpha_{1}\right), h\left(\alpha_{1}\right) \cdot \beta_{1}\right)$ where $\left(f(\alpha)(i)=f(\alpha(i)),(h(\alpha) \cdot \beta)(i)=h(\alpha(i)) \cdot \beta(i)\right.$ for each $i \in \underline{S}$. If $\alpha(i) \neq \alpha_{1}(i)$ for some $i \in \underline{S}$ then we can find $f: \underline{S}^{\circ} \rightarrow \underline{S}^{\circ}$ such that $\left|f\left(\underline{S}^{\circ}\right)\right|=s$ and $f(\alpha(i))=0$ and $f\left(\alpha_{1}(i)\right) \neq 0$ but $f\left(\alpha_{1}(j)\right)=0$ for some $j \in \underline{S}, j \neq i$. Then $(f(\alpha), h(\alpha) \cdot \beta) \neq\left(f\left(\alpha_{1}\right), h\left(\alpha_{1}\right) \cdot \beta\right)$ which is false. Thus $\alpha=\alpha_{1}$. Now choose any $i, j \in \underline{S}$ with $i \neq j$. There exists $f: \underline{S}^{\circ} \rightarrow \underline{S}^{\circ}$ with $\left|f\left(\underline{S}^{\circ}\right)\right|=s$ and $f(i)=f(j) \neq 0$ and so $(h(\alpha(i)) \beta(i))\left(h\left(\alpha_{1}(i)\right) \beta_{1}(i)\right)^{-1}=\left(h(\alpha \overline{(j))} \beta(j))\left(h\left(\alpha_{1}(j)\right) \beta_{1}(j)\right)^{-1}\right.$ that is $h(\alpha(i)) \beta(i) \beta_{1}(i)^{-1} h(\alpha(i))^{-1}=h(\alpha(j)) \beta(j) \beta_{1}(j)^{-1} h(\alpha(j))^{-1}$ for any $h$. Hence for any $c, d \in G$ we have $c \beta(i) \beta_{1}(i)^{-1} c^{-1}=d \beta(j) \beta_{1}(j)^{-1} d^{-1}$ and thus $\beta(i) \beta_{1}(i)=\beta(j) \beta_{1}(j)^{-1}$ for any $i, j \in \underline{S}$. Consequently if $Z(G)$ is the centre of $G$ we have $\beta=\beta_{1}$ if $Z(G)=\{1\}$. Otherwise $\beta_{1}(i)=z \cdot \beta(i)$ for some $z \in Z(G)$ and all $i \in \underline{S}$. Hence, if we regard $Z(G)$ as a subgroup of $S_{S} \circ G$ we see that $\mathscr{G}\left(\bar{e}_{s} N\right)=\left(S_{S} \circ G\right) / Z(G)$. A study of the proof of Theorem $\mathrm{A}$ in the light of these results yields:

Theorem 3. If $N=M_{G}(\Gamma)$ and $\operatorname{dim}_{G} \Gamma=n<\infty$, then

$$
\begin{aligned}
(N, N) \leqslant\left(B(N),\left(\left(S_{n}\right) \circ G\right) / Z(G)\right) \circ\left(B\left(\bar{e}_{n-1} N\right),\right. & \\
& \left.\left(\left(S_{n-1}\right) \circ G\right) / Z(G)\right) \circ \cdots \circ\left(B\left(\bar{e}_{1} N\right),\left(\left(S_{1}\right) \circ G\right) / Z(G)\right) .
\end{aligned}
$$

\section{REFERENCE}

(1) S. Eilenderg. Automata, Languages and Machines. Vol. B. (Academic Press, 1976).

QUEEN'S UNIVERSITY

BELFAST BT7 1NN 